\title{
Long-term results of simplified frozen elephant trunk technique in complicated acute type $A$ aortic dissection: A case-control study
}

(C) The Author(s) 2016

Reprints and permissions: sagepub.co.uk/journalsPermissions.nav DOI: I0.1 I77/I708538II5627728 vas.sagepub.com (S)SAGE

\author{
Misato Kobayashi ${ }^{\prime}$, Lyubov Chaykovska ${ }^{2}$, Bernd van der Loo ${ }^{3}$, \\ Thi Dan Linh Nguyen ${ }^{4}$, Gilbert Puippe ${ }^{4}$, Sacha Salzberg ${ }^{2}$, \\ Hideki Ueda ${ }^{5}$, Francesco Maisano ${ }^{2}$, Felice Pecoraro ${ }^{6}$ and \\ Mario Lachat ${ }^{2}$
}

\begin{abstract}
Aim: To describe the long-term experience of a simplified frozen elephant trunk technique (sFETT) used in complicated acute type $A$ aortic dissection (AAAD) treatment.

Methods and results: Between January 2001 and December 2012, 34 patients (mean age $59.9 \pm 11.0$ years) with complicated AAAD (DeBakey I) underwent an emergency surgery including sFETT. sFETT consisted in gluing the dissected aortic arch wall layers with gelatine-resorcinol adhesive and video-assisted antegrade open arch aortic stent-graft deployment in the arch or proximal descending aorta. In addition to sFETT, the aortic root was addressed with standard techniques. A 30-day mortality was $\mathbf{1 4 . 7 \%}$ (five patients) due to bleeding (I), multiple organ failure (2), and colon ischemia (2). Postoperative morbidity included neurological (2), renal (I) and cardio-pulmonary complications (4), as well as wound infection (I). Mean follow-up was $74.4 \pm 45.0$ months. Actual survival rates were $73.5 \%$ at I year, $70.2 \%$ at 5 years, and $58.5 \%$ at 13 years of follow-up. Six patients died during long-term follow-up from heart failure (I) and unknown reasons (5). Five patients required reoperation for aortic arch (3) or aorto-iliac (2) progression of aneurysm during the mid- and long-term follow-up. The remaining patients showed favorable evolution of the dissected aorta with false lumen occlusion in most cases and stable aortic diameters.

Conclusions: In AAAD patients, sFETT as used in our series is an easy and safe technique to repair the aortic arch. Long-term results after sFETT showed false lumen occlusion and stable aortic diameter in up to 13 years of follow-up.
\end{abstract}

\section{Keywords}

Complicated acute type $\mathrm{A}$ aortic dissection, frozen elephant trunk

\section{Introduction}

Acute type A aortic dissection (AAAD) is the most frequent life-threatening aortic disease. Surgical repair is usually limited to the replacement or repair of the aortic root, replacement of the ascending aorta and, eventually, the aortic arch or a part of it. Unfortunately, any remaining native aortic wall in the aortic arch and descending aorta can undergo degeneration that may require redo procedures with significant complication rates. To prevent such complications, Borst et al. ${ }^{1}$ described the elephant trunk (ET) technique in 1983. The ET technique implies the replacement of the aortic arch with a graft having a free-
'Kawaminami Hospital, Kawaminami Koyu Miyazaki, Japan

${ }^{2}$ Clinic for Cardiovascular Surgery, Zurich University Hospital, Zurich, Switzerland

${ }^{3}$ Clinic of Cardiology, Zurich University Hospital, Zurich, Switzerland

${ }^{4}$ Institute of Diagnostic and Interventional Radiology, Zurich University Hospital, Zurich, Switzerland

${ }^{5}$ Chiba University Hospital Department of Cardiovascular Surgery, Inohana Chuo-ku Chiba, Japan

${ }^{6}$ Vascular Surgery Unit, University Hospital 'P. Giaccone', Palermo, Italy

*Misato Kobayashi and Lyubov Chaykovska contributed equally to this work.

Corresponding author:

Lyubov Chaykovska, Clinic for Cardiovascular Surgery, Zurich University Hospital, Raemistrasse 100, CH-809I Zurich, Switzerland.

Email: lyubov.chaykovska@usz.ch 
floating end (the "elephant trunk") extending from the aortic arch into the descending thoracic aorta. Latter floating graft facilitates secondary descending aortic repair through a lateral thoracotomy. ${ }^{2-4} \mathrm{~A}$ major drawback of the ET technique is that the distal arch anastomosis might be challenging or impossible to seal especially when it is performed in patients with a severe coagulation disorder, as commonly encountered in acute aortic dissections. To face these drawbacks, the frozen elephant trunk (FET) technique was developed and first described in 2003.5 For the FET, a hybrid graft is used. This graft has at its distal end a stentreinforced section that is self-expanding when delivered, and that fixes the graft in the descending aorta without the need to perform an anastomosis. In fact, the stent-reinforced section of the hybrid graft is similar to a self-expanding stent-graft used in endovascular aortic repair (EVAR). The proximal part of the hybrid graft consists of a Dacron graft implanted in the aortic root using standard techniques. The supraaortic vessels may be anastomosed end-to-end to side branches of the graft or as a patch. ${ }^{6-13}$ Unfortunately, some delivery systems are quite cumbersome and deployment of the stented portion of the graft might be imprecise, therefore causing a far more distal landing than intended.

In this article, we describe our long-term experience with a simplified FET technique (sFETT). This technique consists of an implantation of the stent graft into the descending aorta after gluing the layers of the aortic root and aortic arch using a French glue and replacement of the ascending aorta with supraaortic vessels. This is an approach that allows a more accurate landing of the stent graft and was developed in 2001, a time, when FET grafts were not yet available.

\section{Methods}

The study was performed following the principles outlined in the Declaration of Helsinki, the protocol and informed consent was approved by the Institutional Review Board, all subjects gave informed consent. Between January 2001 and December 2012, a total of 34 consecutive patients (22 men and 12 women) with complicated AAAD (DeBakey I) were treated by using sFETT. CT angiography was performed in all patients in order to confirm the diagnosis and to assess the exact extension of the dissection. sFETT was implemented in patients with complicated dissection anatomy such as tight and extensive true lumen channel compression (34) and radiological signs of compromised aortic branch vessels ${ }^{14}$ (renal: 25 and/or visceral: 18 and/or peripheral: 9) with or without clinical symptoms of malperfusion. Dissection extended $>5 \mathrm{~cm}$ into the supraaortic trunks in 19 patients $(56 \%)$ and/or into the visceral and/or the renal vessel in 8 patients (18\%). Overall, dissection extended infrarenally in 33 patients $(97 \%)$. An aneurysm of the aortic arch and/or the descending aorta was detected preoperatively in 19 patients $(56 \%)$. Overall, all patients were considered to be candidates for aortic arch replacement and eventually elephant trunk procedure. Emergent surgery was performed in all patients just after admission.

\section{Operative technique}

Depending on the extension of the dissection and/or the presence of a thrombus and its location and/or extension in the true and/or false lumen, the right or left axillary artery was exposed and cannulated (Figures 1 and 2). A venous cannula was inserted percutaneously into the right atrium from the right common femoral vein in all patients. Cardiopulmonary bypass (CPB) was gradually established, and sternotomy was performed once CPB support was started. Patients' temperature was cooled down to $20-24^{\circ} \mathrm{C}$ before circulatory and cardiac arrest. Generally, the left atrium was cannulated to achieve optimal heart decompression, and the coronary sinus was cannulated for cardioplegia infusion. After circulatory arrest, the aorta was opened and the endoluminal aortic anatomy was carefully inspected. During this time, cerebral perfusion was maintained through the brachiocephalic trunk and/or selective cerebral perfusion through the left carotid and/or the left subclavian artery. After cleaning the dissected arch from any thrombus, the layers were glued together by using French glue, a gelatin-resorcin formalin (GRF) glue (Cardial/BARD, Saint-Etienne, France), and Pruit compliant catheter

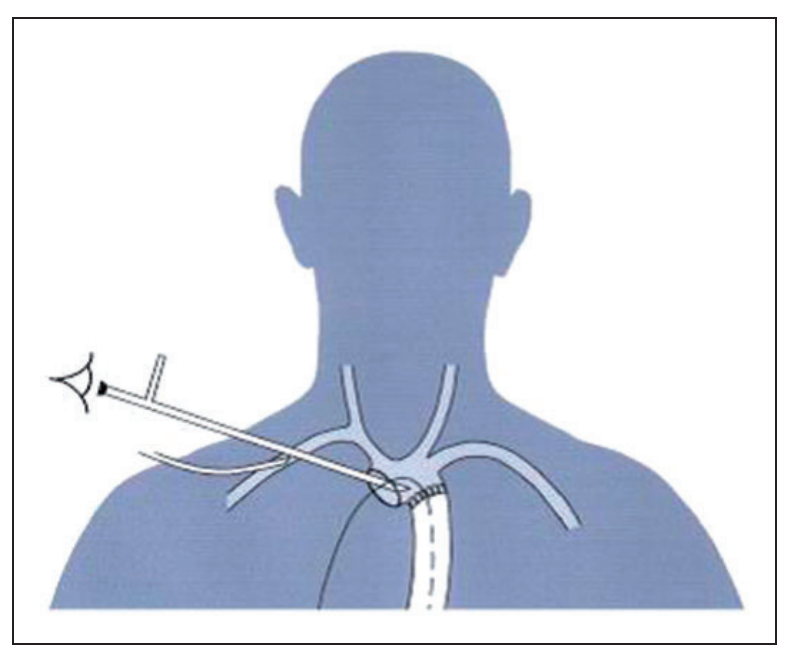

Figure I. Schema of the endoscope assisted stent-graft deployment in circulatory arrest. Antegrade cerebral circulation is performed through the right axillary artery. 


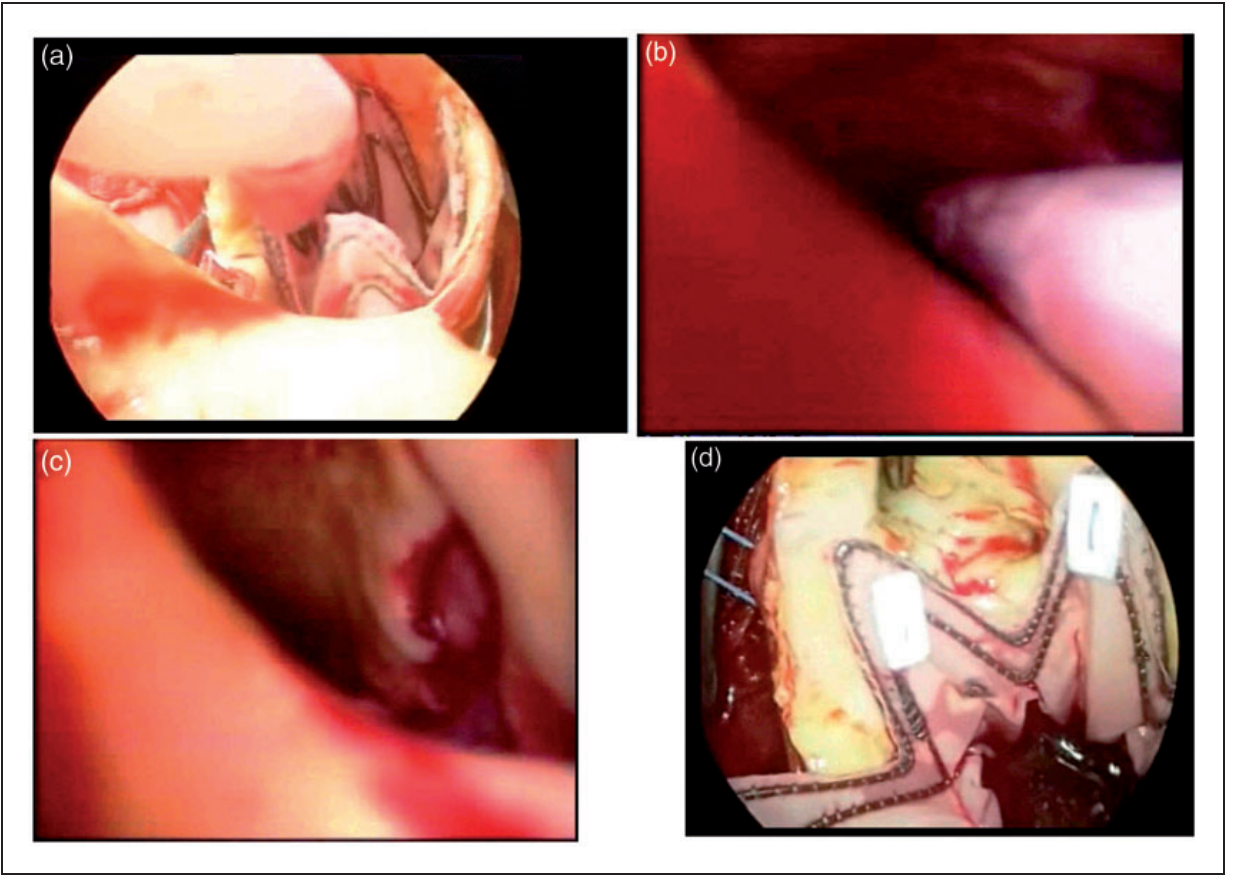

Figure 2. Steps of the video-assisted stent-graft deployment. (a) Inspection of the true channel: Identification of the dissection tear (entry) in the distal arch and of the distal landing zone. (b) Introduction of the stent-graft. Special care is taken of not to pass the stentgraft through the tear and to avoid landing in the false channel. (c) Endoscope controlled stent-graft deployment. (d) Fixation of the stent-graft to the aortic wall just distal to the left subclavian artery.

(LeMaitre, MA, USA) was inflated to achieve full contact between the dissected aortic layers whilst the adhesive was polymerizing. The aortic stent-graft was then inserted into the proximal descending aorta through the open aortic arch over a guide wire. This procedure, especially during the early years, was mostly guided with the support of an endoscopy camera to accurately control the procedure (Figure 1). ${ }^{15}$ In half of the patients, the proximal extremity of the stent-graft was landing distally of the left subclavian artery. In the remaining 17 cases, the stent-graft encroached or covered intentionally the left subclavian artery and/or the left carotid artery in order to exclude an aortic tear located in the aortic arch and subsequent debranching of the covered vessels. Overall, a debranching of the supraaortic trunks was performed in 9 patients. Considering the hypothermic situation and self-expanding properties of Nitinol, the stent-graft was generally not molded with a balloon. In our latter experience, the stent-graft was fixed to the native aortic arch wall by using some pledges-reinforced Ticron sutures (Covidien, Dublin, Ireland). Following this, a polyester graft was sutured in an end-to-end manner to the repaired aortic arch, and systemic perfusion was restored. Finally, the aortic root including the coronary artery ostiums were repaired by using French glue $(n=34)$, and a second graft was sutured to the sino-tubular junction $(n=29)$. Complete aortic root replacement by using biological or mechanical composite graft was performed in 5 patients showing aortic root aneurysm and/or aortic valve disease and/or when root repair was unsuccessful. When two grafts were used, both proximal and distal ascending grafts or distal ascending graft and composite aortic root graft were sutured together.

\section{Stent-graft devices}

Three types of stent-graft were used for sFETT. The TAG stent-graft (WL Gore, AZ, USA) was used in 6 patients $(18 \%)$, the Talent stent-graft (Medtronic, MN, USA) was used in 5 patients $(15 \%)$ and E-Vita stent-graft (Jotec Company, Hechingen, Germany) was used in 23 patients $(67 \%)$. To avoid stentgraft-associated damage of the aortic wall and/or the dissection membrane, the diameter of the stent-graft was not oversized with regard to the native aorta diameter (external aortic diameter at expected landing zone). The Gore TAG mean diameter was $34.5 \pm 3.5 \mathrm{~mm}$, the Talent mean diameter was $34.2 \pm 1.6 \mathrm{~mm}$ and the E-VITA mean diameter was $32.4 \pm 2.9 \mathrm{~mm}$. Stent-graft length was $10 \mathrm{~cm}$ for both the Talent and the Gore stentgraft and $13 \mathrm{~cm}$ for the Evita device. In all patients a single stentgraft was used. 


\section{CT angiography}

Follow-up CT angiography was performed in 28 patients. Maximal aortic diameter was measured preoperatively and during the follow-up at the following levels: aortic root, ascending and descending aorta at the level of pulmonary artery bifurcation, aortic arch or descending aorta at the proximal landing zone of the stent-graft; descending aorta at the level of the distal stent-graft landing zone; visceral aorta at the level of the celiac trunk and the renal arteries, the infrarenal aorta and the common iliac arteries. In patients treated during follow-up with a bifurcated Dacron graft, aortic diameter measurements were taken just before the graft replacement was performed and postoperatively before discharge from the hospital and during the annual follow-up.

\section{Study design and statistics}

Data of all patients treated with sFETT from January 2001 and December 2012 were collected retrospectively according to the ethical standards of our institution. Data analysis included follow-up data of the cohort up to December 2013. Statistical analysis was performed using SPSS 20.0 for Windows (SPSS Inc., Chicago, IL). If not otherwise specified, values are indicated as mean \pm standard deviation (SD). Differences between groups were calculated using the unpaired two-tailed Student's $t$-test. Standard Kaplan-Meier actuarial technique was used to analyze survival. A $p$-value of $<0.05$ was considered significant.

\section{Results}

The patients' mean age was $59.9 \pm 11.0$ years (range 36-80 years) (Table 1 ). All patients initially presented with acute symptoms, mostly chest pain. One patient presented initially unconscious and one patient required a short period of mechanical cardiopulmonary resuscitation. There was no intraoperative death, and 30 days mortality was $14.7 \%$ (five patients). Causes of death were bleeding due to a coagulation disorder (1), multiple organ failure (2), and colon ischemia (2). The patients who died of colon ischemia showed an extensive colon ischemia already on preoperative CT scan. There were neurologic complications in two patients $(5.9 \%)$. Both patients showed hemiplegia postoperatively, and cerebral infarction was confirmed by CT scan. However, one of these patients presented symptoms already preoperatively. One patient had acute renal insufficiency and required hemodialysis; however, he completely recovered. Four patients $(11.8 \%)$ required prolonged intubation and underwent tracheotomy.

Mean follow-up is $49.4 \pm 45.0$ months. Six patients $(18 \%)$ underwent secondary aortic procedures during
Table I. Patients' characteristics.

\begin{tabular}{lc}
\hline Demographic data & \\
- Patients' number & 34 \\
- Mean age (years) & $59.9 \pm 11.0$ \\
- Sex (Male/Female) & $21 / 22$ \\
Comorbidity & \\
- Coronary artery disease & 3 \\
- Cerebral vascular disease & 2 \\
- Chronic obstructive pulmonary disease & 2 \\
Cardiovascular risk factors & \\
- Diabetes mellitus & 2 \\
- Smoking & 10 \\
- Arterial hypertension & 21 \\
Marfan syndrome & 1 \\
Initial symptoms & \\
- Chest pain & 32 \\
- Hemodynamic collapse & 1 \\
- Unconscious & 1 \\
- Hemiplegia & 2 \\
- Peripheral neurology & 4 \\
- Dyspnea & 3 \\
\hline
\end{tabular}

follow-up. There were no complications during or following these procedures. In particular, attention should be drawn to two patients. One patient developed secondary type Ia endoleak necessitating a redo procedure 6 years after AAAD. This patient was treated endovascularly with periscope endograft to the left subclavian artery and T-EVAR landing in Zone 2. One patient developed bilateral iliac artery aneurysms which were addressed 2 years after AAAD by implantation of a bifurcated Dacron graft.

Diameters of the descending aorta and the iliac arteries remained stable during the whole follow-up period (Figure 3). Differences between pre- and postoperative aortic and iliac artery diameters measured at any level were $4.9 \% \pm 21.7 \%, 46.1 \%$ (maximum), $0 \%$ (minimum), and 2.6\% (median). A significant diameter reduction was noted at the level of the aortic root and the ascending aorta; however, this difference reflects the replacement of the diseased aorta, generally an aneurysm, by a smaller sized aortic graft $(p<0.05)$ (Figure 3 ). The false lumen in the aortic arch disappeared postoperatively in 23 patients $(82.1 \%)$ and overall, the diameter of the aortic arch showed a significant reduction $(p<0.05)$ (Figure 3$)$. The false lumen distally from the aortic stent-graft disappeared or significantly decreased in 7 patients $(30 \%)$ (representative examples are shown in Figures 4 and 5). After surgery, there was a significantly lower incidence of aortic branch-vessels compromise in the CT angiography both of the supraaortic vessels $(40.7 \%$ vs. $17.5 \% ; p<0.05)$ 


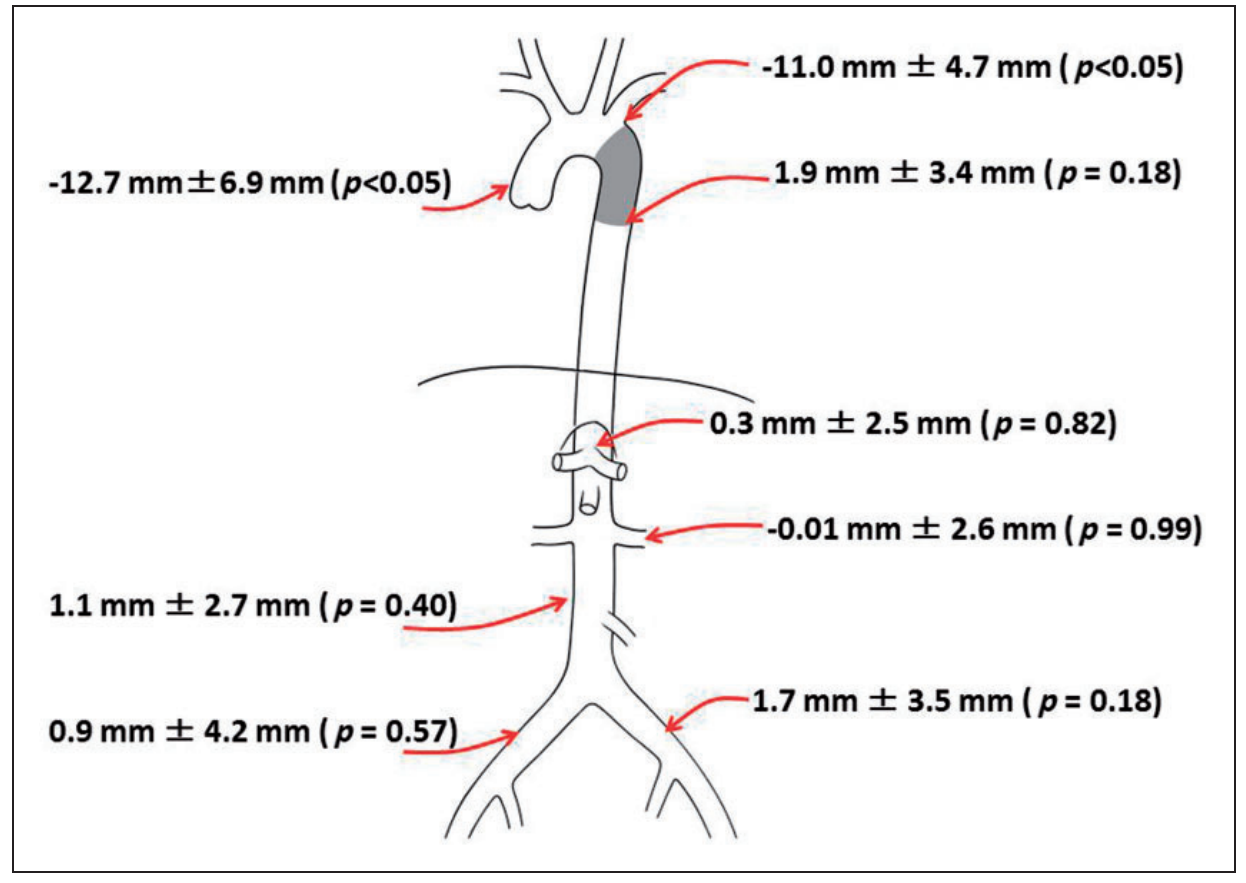

Figure 3. Comparison of pre- and postoperative (last follow-up CTA) diameters at different levels of the aorta. Decrease of diameter at the level of the ascending aorta and the aortic arch are consequences of surgery (i.e. graft replacement).
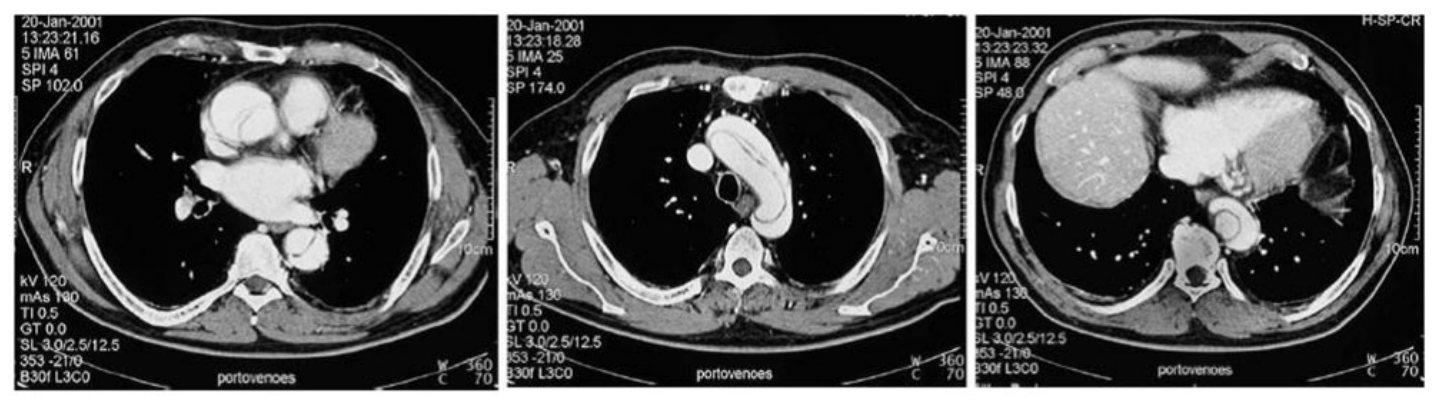

Figure 4. Preoperative $C T$ scan of an AAAD patient. The false lumen extends from the ascending aorta to the abdominal aorta.

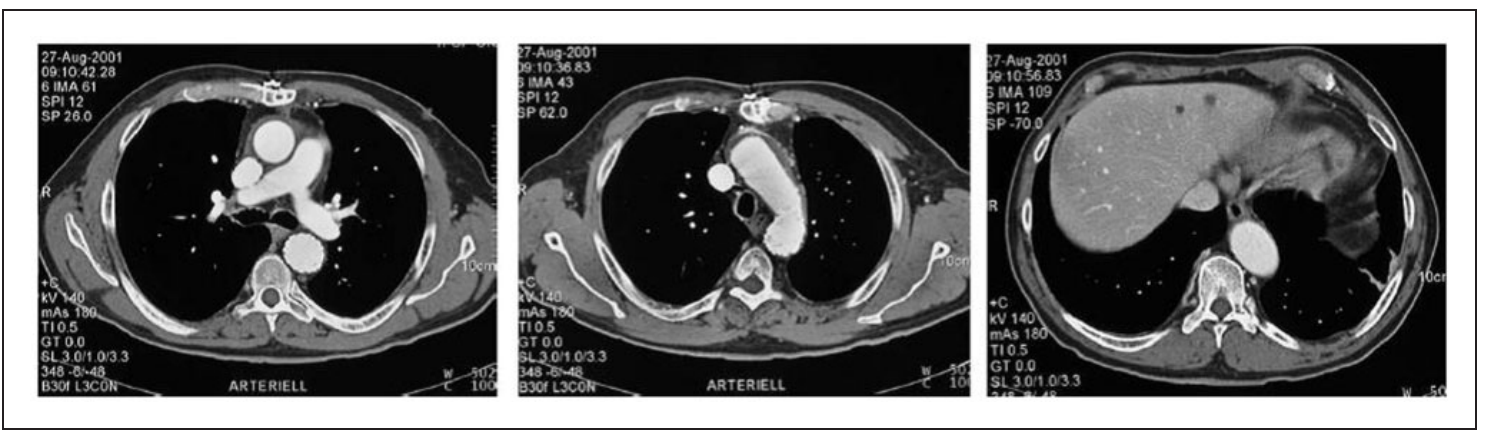

Figure 5. Six months follow-up CT scan of patient of Figure 4. The false lumen disappeared over the entire length after the sFETT. 
Table 2. Dissection and aortic branch vessel compromise.

\begin{tabular}{llll}
\hline & Preoperative & Follow-up & $P$ \\
\hline Dissection & & & \\
- Supraaortic vessels (RSA, RCCA, LCCA, LSA) & $85.2 \%$ & $62.1 \%$ & n. s. \\
- Renovisceral vessels (TC, SMA, RRA, LRA) & $51.9 \%$ & $41.4 \%$ & n. s. \\
- Iliac vessels & $66.7 \%$ & $68.9 \%$ & n. s. \\
Compromised aortic branches & & & \\
- Supraaortic vessels (RSA, RCCA, LCCA, LSA) & $40.7 \%$ & $17.5 \%$ & $<0.05$ \\
- Renovisceral vessels (TC, SMA, RRA, LRA) & $29.6 \%$ & $17.2 \%$ & $<0.05$ \\
- Iliac vessels & $7.4 \%$ & $6.9 \%$ & n. s. \\
\hline
\end{tabular}

RSA: right subclavian artery; RCCA: right common carotid artery; LCCA: left common carotid artery; LSA: left subclavian artery; TC: truncus coeliacus; SMA: superior mesenteric artery; RRA: right renal artery; LRA: left renal artery.

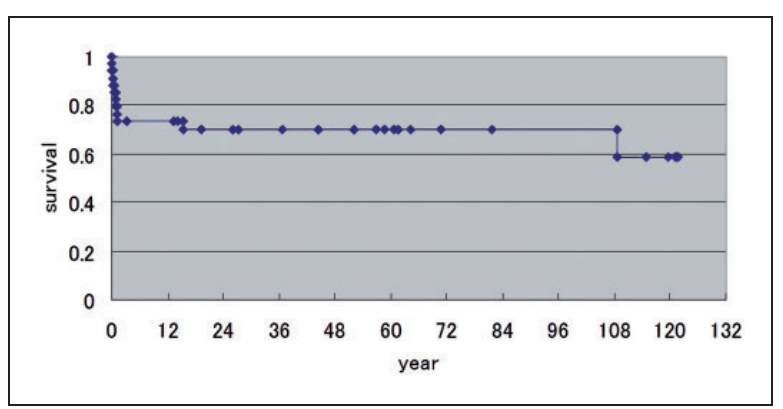

Figure 6. Kaplan-Meier estimate of survival.

and the renovisceral arteries $(29.6 \%$ vs. $17.2 \% ; p<0.05)$ (Table 2).

During long-term follow-up, there were six deaths. One of the patients died from heart failure. The last CT angiography of the other five patients did not show any significant aortic degeneration and, therefore, it may be assumed that the cause of death was not related to an aortic pathology. Survival rate was $73.5 \%$ at 1 year, $70.2 \%$ at 5 years, and $58.5 \%$ at 10 years of follow-up (Figure 6).

\section{Discussion}

The primary purpose of AAAD treatment is to save life and the secondary purpose is to prevent the extension of dissection and the development of an aortic aneurysm which may require an extensive aortic surgery eventually extending to the supraaortic vessels and the descending aorta. Latter procedures, however, have high mortality rates and may cause severe complications such as hemorrhage, cerebral and/or spinal infarction and pulmonary disorders.

Gluing the dissection's layer with adhesives like fibrin glue, French glue or Bioglue is a well-known and effective repair method of the dissected aortic root. ${ }^{16}$ However, this method has drawbacks when used in the dissected aortic arch. In particular, re-dissection and cerebral embolization of glue particles have been described. ${ }^{17}$ In contrast to the aortic root where flow through the false lumen can completely be stopped, the descending aorta mostly remains dissected. As a consequence, retrograde perfusion and re-dissection of the aortic arch might occur soon after restoring aortic perfusion. In some complicated cases of the extended AADs, the true lumen is compressed causing a branch-vessel compromise the abdominal organs and the lower extremities. In order to restore the impaired perfusion, the true lumen of the aorta has to be reopened. In order to achieve this, we developed the sFETT. The sFETT combines gluing the aortic arch and deploying, through the aortic arch, an aortic stent-graft landing in the distal arch and the descending aorta. The stent-graft has different functions. Re-adaptation of the dissected layer is promoted all along the aortic stent-graft down to the mid-descending aorta. It eventually covers and excludes an aortic tear that might be located in the aortic arch or the proximal part of the descending aorta. Thereby, retrograde arch reperfusion and potential cerebral glue embolization is ruled out. Finally, if necessary and in analogy to the frozen elephant trunk technique, a distal extension of the stentgraft in the descending aorta can be achieved by a transfemoral approach. Compared with other repair techniques for AAAD, sFETT is easier and less invasive, especially, a primary aortic arch replacement is not necessary any more. In some cases, it could be eventually combined with a debranching of supraaortic trunks. Besides that, sFETT enables treatment of a tight true lumen channel compression and improves the blood flow of the downstream vessels, thereby preventing a malperfusion. For comparison, 30-day mortality rate of the sFETT in our series of patients with branch-vessel compromise and therefore a high risk of malperfusion is $8 \%$. versus operative mortality of $19 \%$ after FET technique in AAAD patients with 
malperfusion. ${ }^{18}$ A worldwide experience of FET and its variations was recently analyzed in a population of 700 patients including 400 of both acute and chronic dissections, the mean perioperative mortality in dissection patients was $6 \%$ with Ius et al. ${ }^{3}$ A perioperative mortality of $14.7 \%$ in our study using sFETT is at least comparable to these prior reports and can be considered low given the fact that we performed sFETT only in patients with compromised aortic branch vessels a known risk factor for malperfusion and in hospital mortality. $3,19,20$

The impact of the sFETT on the evolution of postoperative aortic dissection is impressive. After sFETT, there was a significantly lower incidence of branchvessel compromise the supraaortic vessels $(40.7 \%$ vs. $17.5 \% ; p<0.05)$ and the renovisceral arteries $(29.6 \%$ vs. $17.2 \% ; p<0.05$ ) (Table 2). In 23 of 28 surviving patients $(82 \%)$, the false lumen in the aortic arch primarily disappeared. This is similar to the outcome of the more complex FET technique with a previously described operative mortality of $8.1 \%{ }^{18}$ The reason for the residual dissection in the aortic arch in five of our patients is not completely clear. Retrograde perfusion from the distal thoracic or abdominal aorta might have maintained the perfusion of the false channel or may have produced a "de novo" dissection in the arch. In our first five cases, the aortic stent-graft was just deployed and not surgically fixed at its proximal landing zone (proximal descending aorta or distal aortic arch). Postoperative CT angiography of these patients showed in some cases a residual perfusion through the false channel. To rule out such leaks, we then started to systematically fix the stent-grafts transparietally at its proximal landing zone, just after its deployment.

We first described sFETT in 2001. ${ }^{15}$ Our long-term sFETT data on 34 patients extend the promising preliminary results of the following series performed by Roselli et al. ${ }^{12}$ These colleagues analyzed early and intermediate outcome of 17 patients operated with sFETT between June 2009 and February 2012. In this study, there were no perioperative deaths, and all patients were still alive at intermediate follow-up. Moreover, the proximal aortic repair was stable in all patients, and the false lumen was thrombosed in the treated segment in $87.5 \%$ of patients, which was similar to our outcome data showing the thrombosis of the false lumen in $82.1 \%$ of the patients.

Simplified FETT is a less invasive and relatively easy and safe technique to repair the aortic arch in patients presenting with complicated AAAD. Our long-term results of up to 13 years follow-up show a false lumen occlusion and stable aortic diameters in most patients. Therefore, sFETT may be considered as a valuable technique for facilitating complex and/or complicated AAAD treatment.

\section{Declaration of conflicting interests}

The author(s) declared no potential conflicts of interest with respect to the research, authorship, and/or publication of this article.

\section{Funding}

The author(s) received no financial support for the research, authorship, and/or publication of this article.

\section{References}

1. Borst HG, Walterbusch $G$ and Schaps D. Extensive aortic replacement using "elephant trunk" prosthesis. Thorac Cardiovasc Surg 1983; 31: 37-40.

2. Heinemann MK, Buehner B, Jurmann MJ, et al. Use of the "elephant trunk technique" in aortic surgery. Ann Thorac Surg 1995; 60: 2-6. (discussion 7).

3. Ius F, Hagl C, Haverich A, et al. Elephant trunk procedure 27 years after Borst: What remains and what is new? Eur J Cardiothorac Surg 2011; 40: 1-11.

4. LeMaire SA, Carter SA and Coselli JS. The elephant trunk technique for staged repair of complex aneurysms of the entire thoracic aorta. Ann Thorac Surg 2006; 81: 1561-1569. (discussion 1569).

5. Karck M, Chavan A, Hagl C, et al. The frozen elephant trunk technique: A new treatment for thoracic aortic aneurysms. J Thorac Cardiovasc Surg 2003; 125: $1550-1553$.

6. Hoffman A, Parker JA, Raweh A, et al. Restoration of the thoracic aorta in Type A dissection with hybrid prosthesis. Asian Cardiovasc Thorac Ann 2011; 19: 123-127.

7. Uchida N, Shibamura H, Katayama A, et al. Total arch replacement with an open stent graft for acute type A aortic dissection: fate of the false lumen. Eur $J$ Cardiothorac Surg 2009; 35: 83-88.

8. Pacini D, Tsagakis K, Jakob H, et al. The frozen elephant trunk for the treatment of chronic dissection of the thoracic aorta: A multicenter experience. Ann Thorac Surg 2011; 92: 1663-1670. (discussion 1670).

9. Gorlitzer M, Weiss G, Moidl R, et al. Repair of stent graft-induced retrograde type A aortic dissection using the E-vita open prosthesis. Eur J Cardiothorac Surg 2012; 42: 566-570.

10. Di Bartolomeo R, Di Marco L, Armaro A, et al. Treatment of complex disease of the thoracic aorta: The frozen elephant trunk technique with the E-vita open prosthesis. Eur J Cardiothorac Surg 2009; 35: 671-675. (discussion 675-676).

11. Uchida N, Katayama A, Tamura K, et al. Long-term results of the frozen elephant trunk technique for extended aortic arch disease. Eur J Cardiothorac Surg 2010; 37: 1338-1345.

12. Roselli EE, Rafael A, Soltesz EG, et al. Simplified frozen elephant trunk repair for acute DeBakey type I dissection. J Thorac Cardiovasc Surg 2013; 145: S197-201.

13. Kouchoukos NT, Mauney MC, Masetti P, et al. Optimization of aortic arch replacement with a onestage approach. Ann Thorac Surg 2007; 83: S811-814. (discussion S824-831). 
14. Williams DM, Lee DY, Hamilton $\mathrm{BH}$, et al. The dissected aorta: part III. Anatomy and radiologic diagnosis of branch-vessel compromise. Radiology 1997; 203: 37-44.

15. Lachat M. Introduction to antegrade endovascular descending thoracic aortic repair. In: HeartLab international workshop, Geneva, Switzerland, 24-28 October 2001, p. 7.

16. Borst $\mathrm{HG}$, Laas $\mathrm{J}$ and Buhner B. Efficient tissue gluing in aortic dissection. Eur J Cardiothorac Surg 1994; 8: 160-161.

17. Carrel T, Maurer M, Tkebuchava T, et al. Embolization of biologic glue during repair of aortic dissection. Ann Thorac Surg 1995; 60: 1118-1120.
18. Ma WG, Zheng J, Zhang W, et al. Frozen elephant trunk with total arch replacement for type A aortic dissections: Does acuity affect operative mortality? $J$ Thorac Cardiovasc Surg 2014; 148: 963-970; discussion 970-972.

19. Trimarchi S, Nienaber CA, Rampoldi V, et al. Contemporary results of surgery in acute type A aortic dissection: The International Registry of Acute Aortic Dissection experience. J Thorac Cardiovasc Surg 2005; 129: 112-122.

20. Nagamine $\mathrm{H}$, Ueno $\mathrm{Y}$, Ueda $\mathrm{H}$, et al. A new classification system for branch artery perfusion patterns in acute aortic dissection for examining the effects of central aortic repair. Eur J Cardiothorac Surg 2013; 44: 146-153. 